\title{
Complex visual hallucinations in mentally healthy people
}

\author{
Alucinações visuais em pessoas normais \\ Marco A. Lana-Peixoto
}

Departamento de Neuro-

Oftalmologia, Faculdade de

Medicina Universidade Federal de

Minas Gerais, Belo Horizonte MG,

Brazil.

Correspondence:

Marco Aurélio Lana-Peixoto; Rua Padre Rolim, 769/Conjunto 1301

Santa Efigênia; 30130-090, Belo

Horizonte MG, Brasil;

E-mail: marco.lanapeixoto@gmail.com

Conflict of interest:

There is no conflict of interest to declare.

Received 20 March 2014

Accepted 02 April 2014
“... I know a respectable man, full of health, of innocence, judgement and memory, who, completely watchful and independently from all outside influences, perceives from time to time, in front of him, Figures of Men, Women, Birds, of buildings..."

Charles Bonnet, $1760^{1}$

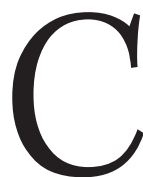

omplex visual hallucinations are still an intriguing phenomenon that occurs in a wide variety of circumstances, from normally circadian states to severe neurological and psychiatric conditions.

Up to one third of normal individuals experience visual hallucinations prior to falling asleep or immediately following arousal. These hypnagogic and hypnopompic hallucinations occur in a variable frequency, last few seconds to minutes, and may be as simple as spots of lights, lines or geometric patterns to complex images such as faces, figures of real or bizarre humans or animals, objects or scenery. Associated emotional reaction to these complex images may be neutral, pleasant, or sometimes extremely frightening ${ }^{2}$.

In addition to these physiological sleep-related hallucinations, unreal visual perceptions may be associated with a variety of abnormal conditions such as sight deprivation, some paroxysmal disorders as narcolepsy-cataplexy syndrome, migraine and partial epilepsy; brainstem and thalamic lesions; Parkinson's disease and Lewy body dementia; affective disorders, schizophrenia and other psychoses; effects of hallucinogenic drugs; and alcohol, barbiturate and benzodiazepine withdrawal ${ }^{2,3,4,5,6,7,8}$. As perceptual disturbances are a hallmark of severe mental disorders, complex visual hallucinations are a common finding in psychiatric setting. In schizophrenia and other psychoses complex visual hallucinations accompany auditory hallucinations, and in contrast to those occurring in mentally healthy people they are often present throughout waking hours, and have less insight and more paranoia. In most patients, a careful medical history and physical examination will provide key elements to initially distinguish mentally-sick from mentally-healthy individuals, and then attain, in both situations, accurate diagnosis underlying the defective visual perception.

Two different pathophysiological mechanisms to explain the phenomenology of the visual hallucinations as a result of a wide variety of conditions have been proposed. Visual hallucinations in the context of visual sensory deprivation, migraine and epilepsy seem to be caused by direct excitation of the visual cortex or by de-afferentiation. On the other hand, visual hallucinations related to narcolepsy, peduncular lesions, Parkinson's disease and Lewy body dementia, Alzheimer's disease, delirium, schizophrenia and those occurring as drug effects would be caused by pathology and dysfunction of the ascending brainstem cholinergic pathways ${ }^{9}$.

In this issue of Arquivos de Neuro-Psiquiatria, Vale et al..$^{10}$, describe a series of eight patients from their hospital Low Vision Department who presented complex visual hallucinations. All patients had poor vision as a result of different eye pathologies. Their unrevealing medical history and neurologic examination, including a battery of cognitive tests, ruled out a list of differential diagnosis as alternative causes for visual hallucinations, and then established the diagnosis of Charles Bonnet syndrome (CBS). This report emphasizes that practitioners need to assess mental and cognitive functions in patients with complex visual hallucinations to rule out psychiatric and cognitive disorders. In addition to 
CBS, complex visual hallucinations may be experienced by mentally healthy individuals with different neurologic conditions, such as narcolepsy-cataplexy syndrome, peduncular hallucinosis, basilar and familial hemiplegic migraine, and partial epilepsy ${ }^{2,11}$.

Visual hallucinations in mentally healthy people with visual impairment has been known as CBS, since the Swiss naturalist described the case of his grandfather in 1760 and George de Morsier introduced the eponym in $1967^{1,12}$. Ironically, Bonnet himself developed the syndrome ${ }^{13}$. Although their pathophysiology is not completely clear visual hallucinations in CBS are ascribed to de-afferentiation of the visual cortex which causes its endogenous activation and spontaneous firing ${ }^{14,15}$. Hallucinations more often are formed and include images of animals, people or scenes in the presence of a clear sensorium. They are more frequent in the night, poor light, or personal isolation. Sometimes, Lilliputian hallucinations, defined as a visual phenomenon in which a patient views miniature people in lines or groups, often undertaking unusual postures or actions and accompanied by wonder rather than terror are reported by patients ${ }^{16}$.

Narcolepsy-cataplexy syndrome is characterized by excess daytime sleepiness, sleep paralysis, cataplexy and hypnagogic hallucinations. The disease is most commonly constitutional and secondary to the specific loss of hypocretin-producing neurons in the lateral hypothalamus, but acquired cases with lesions in the brainstem may occur with features similar to those found in peduncular hallucinosis ${ }^{5,17}$. Hypnagogic visual hallucinations are associated with drowsiness, lasting seconds to minutes. However, as in CBS insight is well preserved.

The term peduncular hallucinosis has been used to describe complex hallucinations associated with lesions in the brainstem and thalamus ${ }^{5}$. Patients have no alteration of consciousness but abnormal brainstem symptoms may be present. Although the main cause is infarction, hemorrhage and infections have also been found. Hallucinations generally start few days after the event, last minutes but may persist for several hours, to subside a few weeks later. They frequently are absent during the daytime but recur each evening. Patients have insight into the hallucinations and cope with them well. In peduncular hallucinosis, as in CBS, Lilliputian hallucinations are not rare.

Migraine most frequently causes simple visual hallucinations, but complex visual hallucinations may occur in basilar migraine and familial hemiplegic migraine. In contrast to hallucinations in migraine aura, complex visual hallucinations in basilar migraine appear at a late stage of an attack usually following symptoms of brainstem and cerebellar dysfunction or at recovery from coma. They may last hours or days and insight is preserved ${ }^{2}$.

Partial epilepsy is also a cause of episodic complex visual hallucinations ${ }^{2}$. Pathological discharges from the visual cortical areas, mainly from posterior parietal and temporal association cortex, may produce brief, recurrent and stereotyped visual hallucinations. The visual phenomena may be the only manifestation of the ictus or they may be accompanied by disturbed awareness, automatisms, sensory and motor abnormalities. The electroencephalogram shows bursts of epileptiform activity over the posterior parietal and temporal association cortex.

As knowledge of the visual hallucinations remains most fragmentary and elusive, clinicians should be aware that a comprehensive evaluation of their patients may provide them with tools to better understand the phenomenon and reduce their burden.

\section{References}

1. Bonnet C. Essai Analytique sur les Facultés de L’Âme. Copenhagen: Ferres \& Philibert; 1760:426-429.

2. Manford M, Andermann F. Complex visual hallucinations: clinical and neurobiological insights. Brain 1998;121:1819-1840.

3. Teunisse RJ, Cruysberg JR, Hoefnagels WH, Verbeek AL, Zitman FG. Visual hallucinations in psychologically normal people: Charles Bonnet's syndrome. Lancet 1996;347:794-797.

4. Lowe GR. The phenomenology of hallucinations as an aid to differential diagnosis. Br J Psychiatry 1973;123:621-633.

5. Kolmel HW. Peduncular hallucinations. J Neurol 1991;238:457-459.

6. Sanchez-Ramos JR, Ortoll R, Paulson GW. Visual hallucinations associated with Parkinson disease. Arch Neurol 1996;53:1265-1268.

7. Mueser KT, Bellack AS, Brady EU. Hallucinations in schizophrenia. Acta Psychiatr Scand 1990; 82:26-29.

8. Rosenthal SH. Persistent hallucinosis following repeated administration of hallucinogenic drugs. Am J Psychiatry 1964;121:238-244.

9. ffytche DH. Visual hallucinations and the Charles Bonnet syndrome. Cur Psychiat Rep 2005; 7:168-179.
10. Vale TC, Fernandes LC, Caramelli P. Charles Bonnet syndrome: characteristics of its visual hallucinations and differential diagnosis. Arq Neuropsiquiatr 2014;72:333-336.

11. Mocellin R, Walterfang M, Velakoulis D. Neuropsychiatry of complex visual hallucinations. Aust N Zeal J Psychiatry 2006;40:742-751.

12. de Morsier G. Le syndrome de Charles Bonnet. Ann Med Psych 1967;128:677-702.

13. Fernandez A, Lichtshein G, Vieweg W. The Charles Bonnet syndrome: a review. J Nerv Ment Dis 1997;185:195-200.

14. Schultz G, Melzack R. The Charles Bonnet syndrome: 'phantom visual images'. Perception 1991;20:809-825.

15. Cogan DG. Visual hallucinations as release phenomena. Graefe's. Arch Clin Exp Ophthalmol 1973;188:139-150.

16. Leroy R. The syndrome of Lilliputian hallucinations. J Nerv Ment Dis 1922;56:325-333.

17. Hishikawa Y, Nan'no H, Tachibana M, Furuya E, Koida H, Kaneko Z. The nature of sleep attack and other symptoms of narcolepsy. Electroencephalogr Clin Neurophysiol 1968;24:1-10. 